\title{
Carbon nanotube film replacing silver in high-efficiency solid-state dye solar cells employing polymer hole conductor
}

\author{
Kerttu Aitola $^{1}$ - Jinbao Zhang ${ }^{1} \cdot$ Nick Vlachopoulos $^{2}$ - Janne Halme ${ }^{3}$. Antti Kaskela ${ }^{3}$. \\ Albert G. Nasibulin ${ }^{3,4}$ • Esko I. Kauppinen ${ }^{3}$ - Gerrit Boschloo ${ }^{1}$ • Anders Hagfeldt ${ }^{1,2}$
}

Received: 23 January 2015 /Revised: 17 June 2015 / Accepted: 21 June 2015 /Published online: 2 July 2015

(C) Springer-Verlag Berlin Heidelberg 2015

\begin{abstract}
A semitransparent, flexible single-walled carbon nanotube (SWCNT) film was efficiently used in place of evaporated silver as the counter electrode of a poly(3,4-ethylenedioxythiophene) polymer-based solidstate dye solar cell (SSDSC): the solar-to-electrical energy conversion efficiency of the SWCNT-SSDSC was $4.8 \%$ when it was $5.2 \%$ for the Ag-SSDSC. The efficiency difference stemmed from a $0.1-\mathrm{V}$ difference in the open-circuit voltage, whose reason was speculated to be related to the different recombination processes in the two types of SSDSCs.
\end{abstract}

Keywords Dye-sensitized solar cell · Polymer hole-conducting medium .

Poly(3,4-ethylenedioxythiophene) · Counter electrode ·

Carbon nanotube film

Kerttu Aitola

kerttu.aitola@kemi.uu.se

Nick Vlachopoulos

nikolaos.vlachopoulos@epfl.ch

1 Department of Chemistry - Ångström Laboratory, Physical Chemistry, Uppsala University, P.O. Box 523,

75120 Uppsala, Sweden

2 Institute of Chemical Sciences and Engineering, École Polytechnique de Fédérale de Lausanne, EPFL SB ISIC LSPM, CH G1 523, Chemin des Alambics, Station 6, CH-1015 Lausanne, Switzerland

3 Department of Applied Physics, Aalto University School of Science, P.O. Box 15100, 00076 Aalto, Finland

4 Skolkovo Institute of Science and Technology, 100 Novaya Street, Skolkovo, Russia

\section{Introduction}

The dye solar cell [1] (DSC) is an interesting emerging technology for low-cost production of photovoltaic devices. However, one of its possible downsides in the most common configuration is the use of volatile liquid electrolytes, which may cause long-term stability problems [2] and set strict criteria for the cell sealing. An alternative to liquid electrolytes as chargetransport media is provided by solid-state hole conductors, such as 2,2'7,7'-tetrakis-( $N, N$-di-p-methoxyphenylamine)-9, 9 '-spirobifluorene [3], other small molecule hole conductors [4], and poly(3,4-ethylenedioxythiophene) (PEDOT) [5], which are thought to be more stable than liquid electrolytes.

Conjugated polymer-based hole-transport media, such as PEDOT, are attractive due to the low cost of the starting material, relatively easy manufacturing, flexibility, and semitransparency. The efficiency of the PEDOT-based solid-state DSC (SSDSC) was hitherto limited due to the poor penetration of the spin-coated PEDOT in the mesoporous $\mathrm{TiO}_{2}$ pores, until Liu et al. obtained a high power conversion efficiency of $6.1 \%$ with a SSDSC utilizing in situ photoelectropolymerized PEDOT as charge-transport medium, using the bis-EDOT dimer as precursor and an organic dye with matching energy levels as sensitizer for the bis-EDOT oxidation [6]. Later, they reported a $7.1 \%$ efficiency using monochromatic light in the polymerization [7].

The PEDOT-SSDSCs, like all SSDSCs, require a conducting silver or gold layer counter electrode (CE), or cathode, since the lateral conductivity of the hole-transport layer is not sufficient as such for the solar cell purposes; however, the CE metals may suffer from stability problems due to corrosion. Carbon nanotube films are an interesting alternative to conducting and catalytic layers utilized in solar cells: they are potentially inexpensive, very flexible, and semitransparent, to mention a few of their alluring properties. Single- 
walled carbon nanotube (SWCNT) films have superior conductivity versus transparency properties compared to other nanotube film types [8]. Moreover, SWCNT films can be deposited on different surfaces by a simple press-transfer from a filter paper [8], rendering them suitable for industrial roll-toroll manufacturing methods.

SWCNT and SWCNT composite films have been used as hole-conducting electrodes in bulk heterojunction polymer solar cells [9-11] but not in SSDSCs utilizing conductive polymer-based hole conductor medium. In liquid DSCs, random network SWCNT films have been utilized on conductive $[12,13]$ and non-conductive substrates $[14,15]$ as counter electrodes. We have previously suggested that the iron nanoparticles in the SWCNT films, which are introduced there during the SWCNT synthesis, have to be electrochemically removed from the films in order to prevent their adverse effect on the photoelectrode current production; this effect is probably due to the redox mediator oxidizing the iron $[14,15]$. In the lead perovskite-absorber solar cells [16], SWCNT films have been used successfully as a replacement for the conducting silver or gold layer [17] and in composite hole conductors $[18,19]$. Snaith et al. state that the efficient performance of the devices is related to the fact that the SWCNT film is a better selective contact than $\mathrm{Ag}$ and $\mathrm{Au}[18,19]$.

Carbon nanoparticle-based CEs have been used in the monolithic SSDSCs $[20,21]$ and also in the conventional PEDOT-SSDSC [22].

In this work, we tested whether a press-transferred random network SWCNT film would function as the conducting counter electrode layer in a PEDOT-based SSDSC in place of the usual silver or gold layer. When SWCNT films are used in the standard liquid-based DSCs, the iron nanoparticles present from the SWCNT synthesis have to be removed from the films, as previously mentioned. In the present work, it was anticipated that in the case of SSDSCs, this treatment would not be necessary. The SWCNT film was compared with an evaporated silver layer CE.

\section{Experimental}

The fluorine-doped tin oxide (FTO) conducting glass substrates (Pilkington, TEC-15) were first cut to the DSC dimensions; then, one edge of the glass piece, where the electrical CE silver contact would later be applied, was etched with zinc powder and $4 \mathrm{M} \mathrm{HCl}$ (aq.). Subsequently, the substrates were washed in detergent solution, in acetone and in ethanol in an ultrasonic bath, each step lasting $1 \mathrm{~h}$. The recombinationblocking $\mathrm{TiO}_{2}$ underlayer was prepared with spray pyrolysis of a titanium diisopropoxidebisacetylacetonate solution on the FTO glass substrate kept on a hot-plate at $500{ }^{\circ} \mathrm{C}$. This procedure was applied ten times. The main dye-absorbing 5- $\mu \mathrm{m}$ mesoporous $\mathrm{TiO}_{2}$ layer was deposited by doctor blading of
$\mathrm{TiO}_{2}$ paste (Dyesol DSL 18 NR-T) on the underlayer-coated FTO glass substrate. Subsequently, the electrodes were sintered in an oven at $450{ }^{\circ} \mathrm{C}$ in air for $30 \mathrm{~min}$. The $\mathrm{TiCl}_{4}$ after-treatment, performed in order to increase the real surface of the $\mathrm{TiO}_{2} /$ FTO layer, was carried out by immersing the electrode in $40 \mathrm{mM} \mathrm{TiCl}_{4}$ in water at $70{ }^{\circ} \mathrm{C}$ for $30 \mathrm{~min}$, followed by a new sintering step at $450{ }^{\circ} \mathrm{C}$ for $30 \mathrm{~min}$. The photoelectrodes (PE) were coated with the LEG4 organic dye [23] by immersing overnight in $0.3 \mathrm{mM}$ ethanolic dye solution and rinsed with ethanol before the polymerization.

The photoelectropolymerization was conducted in 10 mMbis-EDOT (Kaïron Kem) and 0.1 M1-ethyl-3methylimidazolium bis(trifluoromethylsulfonyl)imide in acetonitrile (ACN) by imposing a constant current of $8 \mu \mathrm{A} / \mathrm{cm}^{2}$ (chronopotentiometry) with a white LED lamp providing an illumination intensity of about $4 \mathrm{~mW} / \mathrm{cm}^{2}$. The polymerization time was set to $2000 \mathrm{~s}$. Afterward, the PEs were rinsed with ethanol and dried in air.

The SWCNTs for the CE were prepared by the aerosol gas phase method described in [8]. In brief, a ferrocene-containing $\mathrm{CO}-\mathrm{CO}_{2}$ gas mixture $\left(\mathrm{CO}: \mathrm{CO}_{2}\right.$ ratio $\left.99: 1\right)$ is directed to a tube furnace kept at around $880{ }^{\circ} \mathrm{C}$. The ferrocene decomposes in the furnace and forms iron nanoparticles, which act as catalysts for the SWCNT growth. The SWCNTs form bundles in the gas phase, and the bundles are directed out of the reactor and collected on a porous filter paper, on which the film is formed. The film thickness, transparency, and sheet resistance $\left(R_{\mathrm{SH}}\right)$ depend on the collection time of the SWCNT bundles.

The SWCNT film was transferred on the PEDOT layer by a simple press-transfer method, described in [8]. The SWCNT film on the filter paper was applied upside down on the PEDOT layer, and the filter paper was pressed with a finger, so that the SWCNT film was detached from the filter paper and attached on the PEDOT layer.

The silver counter electrode was prepared by evaporating a 200-nm Ag film on the PEDOT layer under vacuum in a Leica EM MED020 evaporator.

Finally, the cells were treated by applying a few drops of $0.2 \mathrm{M}$ 4-tert-butylpyridine (TBP) and $0.025 \mathrm{M}$ lithium bis(trifluoromethylsulfonyl)imide (LiTFSI) in ACN on them and letting the ACN evaporate. The Li-TBP after-treatment is essential for improving the power conversion performance of the solar cells. The SWCNT film densification, which is usually carried out with ethanol [8], was done with the TBP and LiTFSI-containing ACN solution simultaneously with the aforementioned after-treatment, in order to reduce the number of the cell manufacturing steps and so lessen the risk of dye and PEDOT dissolution after the photoelectropolymerization. Some silver paint was applied on the SWCNT film at the edge of the cell, where the FTO glass was etched, to form an electrical contact for the alligator clips of the measurement devices. The SWCNT-PEDOT cell manufacturing steps are shown in Scheme 1. 
1) Dyed photoelectrode

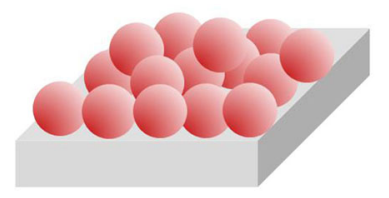

\section{3) SWCNT film transfer}

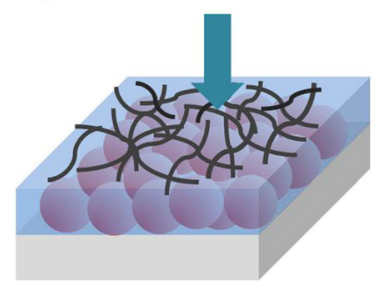

Scheme 1 A schematic image of the cell manufacturing process for the PEDOT-SWCNT cells. The steps are the same for the PEDOT-Ag cells apart from step 3, in which the Ag evaporation is carried out instead

The SWCNT film optical transmittance spectrum was measured on a film transferred on a polyethylene terephthalate (PET) plastic substrate and densified with ethanol, since this is the standard way for conducting this measurement $[8,14]$. The spectrum was obtained with a dual-beam spectrophotometer (Lambda 950, Perkin-Elmer). The sheet resistance measurement was carried out both on a pristine SWCNT film and a film treated with small amount of drop-cast TBP- and LiTFSI-containing ACN solution, both films on PET substrate, in order to see if the LiTFSI and TBP have an effect on the conductive properties of the films. The sheet resistance was measured with an automatic mapping four-point probe system (CMT-SR2000N, Materials Development Corporation). The scanning electron microscopy (SEM) images of the SWCNT film were obtained with a JSM-7500F scanning electron microscope (JEOL Ltd.).

The solar cells were characterized by performing currentpotential $(I-V)$ measurements under illumination by a solar simulator (Newport, model 91160) equipped with a Keithley 2400 source meter and providing a $1000-\mathrm{W} / \mathrm{m}^{2}$ AM $1.5 \mathrm{G}$ illumination intensity, measured by means of a certified reference solar cell (Fraunhofer ISE). The $I-V$ measurements were also carried out at lower light intensities by applying filters in front of the aperture of the solar cell sample holder. The lightintensity dependence measurement was carried out on the same day as the cells were assembled, but the final full sun efficiency measurements were performed on the following day, since it is known that the efficiency improves for these types of cells during the first couple of days. The cells were additionally characterized by optoelectronic "tool-box" measurements, in which electron lifetimes in the $\mathrm{TiO}_{2}$ film were determined by monitoring photovoltage transients at different light intensities upon applying a small square wave modulation to the base light intensity. The toolbox consists of a white LED (Luxeon Star $1 \mathrm{~W}$ ) light source and a 16-bit resolution digital acquisition board, a current amplifier, and a custommade electromagnetic switch system.

\section{Results and discussion}

A typical PEDOT photoelectropolymerization curve is shown in Fig. 1. Chronopotentiometry was chosen as the polymer formation method, since controlling the polymerization potential allows avoiding polymer overoxidation and forming polymer at the sites of the substrate, where the recombinationblocking layer has a possible pinhole and the FTO glass substrate is exposed [24]. In the very beginning of the polymerization, the voltage is high because the polymer has to be formed on a foreign substrate, where there is no polymer. When the surface becomes more covered with the polymer, the voltage begins to drop. As the polymerization proceeds, the voltage starts increasing again as the formation of polymer becomes more difficult in the pores of the $\mathrm{TiO}_{2}$. According to our practical experience, the voltage does not exceed $0.5 \mathrm{~V}$ in a chronopotentiometry experiment resulting in a good quality polymer layer.

The $R_{\mathrm{SH}}$ of the pristine SWCNT film was $20 \Omega / \mathrm{sq}$ and that of the film densified with the LiTFSI and TBP in ACN solution $17.8 \Omega /$ sq. The lowering of the $R_{\mathrm{SH}}$ value in the densification process is a typical phenomenon for these type of films [8], and it can be seen from the values that the TBP and LiTFSI do not have a notable effect on the $R_{\mathrm{SH}}$. The optical transmittance at $550 \mathrm{~nm}$ was about $4 \%$, rendering the film still slightly transparent. The aerosol-produced SWCNT film is of good quality: the nanotubes are long and defect-free $[8,14]$,

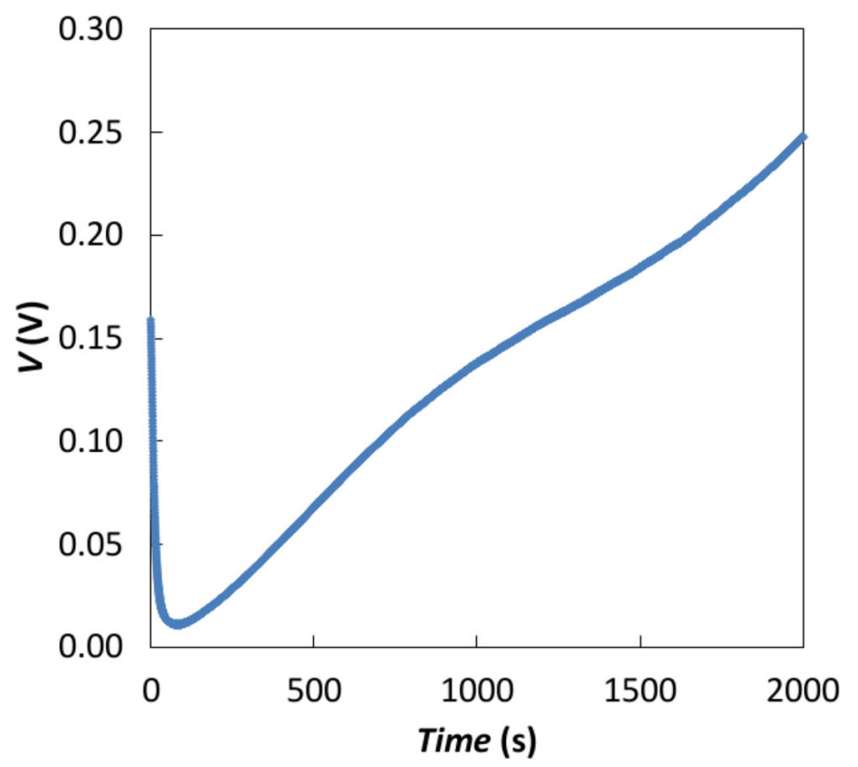

Fig. 1 A typical in situ photoelectropolymerization curve (chronopotentiometry) of the bis-EDOT dimer resulting in a PEDOT polymer hole conductor layer 
which means that good conductivity versus SWCNT film thickness (transparency) values can be achieved, since the film conductivity depends on the quality and length of the nanotubes. A SEM image of the random network type film is shown in Fig. 2. The iron nanoparticles present in the films from the synthesis are shown as bright spots in the image.

The SWCNT film transfer on the PEDOT layer in the solar cell manufacturing was performed without problems, and the SWCNT film densification, which is usually carried out with ethanol [8], worked well with the TBP and LiTFSI in ACN solution; thus, it can be concluded that the densification can be also carried out with solvents other than ethanol. The ethanol was replaced by the ACN solution in order to reduce the number of the solution processing steps in the DSC preparation and also to reduce the risk of possible PEDOT or dye dissolution.

The $I-V$ measurement results are shown in Fig. 3 and Table 1. The average efficiency of the SWCNT-PEDOT cells and the Ag-PEDOT cells was 4.8 and $5.2 \%$, respectively. The difference in the efficiency arises mainly from the difference in the open-circuit voltage $\left(V_{\mathrm{OC}}\right)$, which was $0.7 \mathrm{~V}$ for the SWCNT-PEDOT cells and $0.8 \mathrm{~V}$ for the Ag-PEDOT cells; the cause of this difference will be discussed later in this article. The iron nanoparticles present in the SWCNT films did not cause immediate deterioration in the photocurrent production in the SSDSCs as they do in the liquid DSCs; in the latter case, it is thought that the oxidized form of the redox mediator in solution oxidizes the Fe nanoparticles, with deleterious side-effects to the solar cell performance $[14,15]$. The microscopic reason for this phenomenon is not totally clear, but it can be overcome by a thorough electrochemical removal of the Fe impurity from the films [14]. As can be seen from the very similar current values of the two types of cells, it can be concluded that the Fe removal might not be necessary in this type of a cell configuration, and omitting the electrochemical

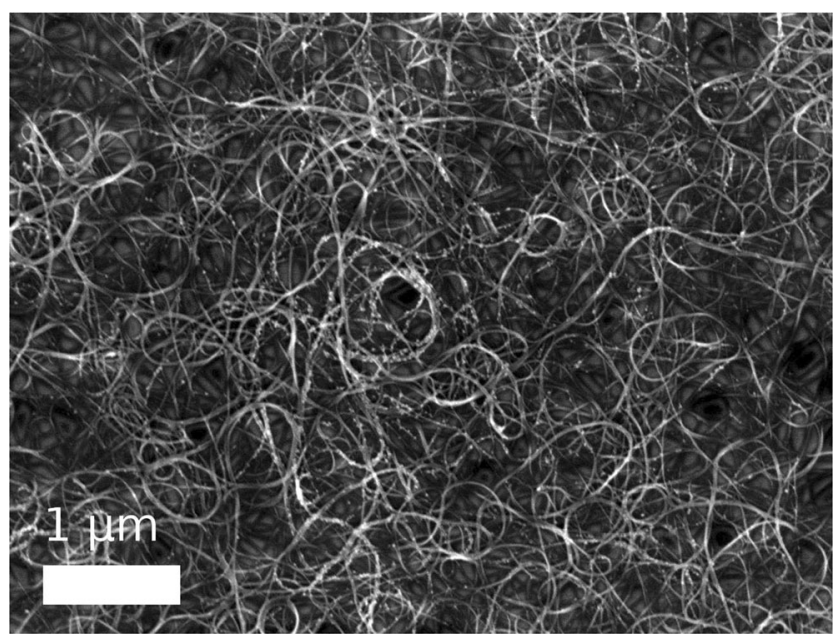

Fig. 2 A scanning electron microscopy image of the single-walled carbon nanotube film

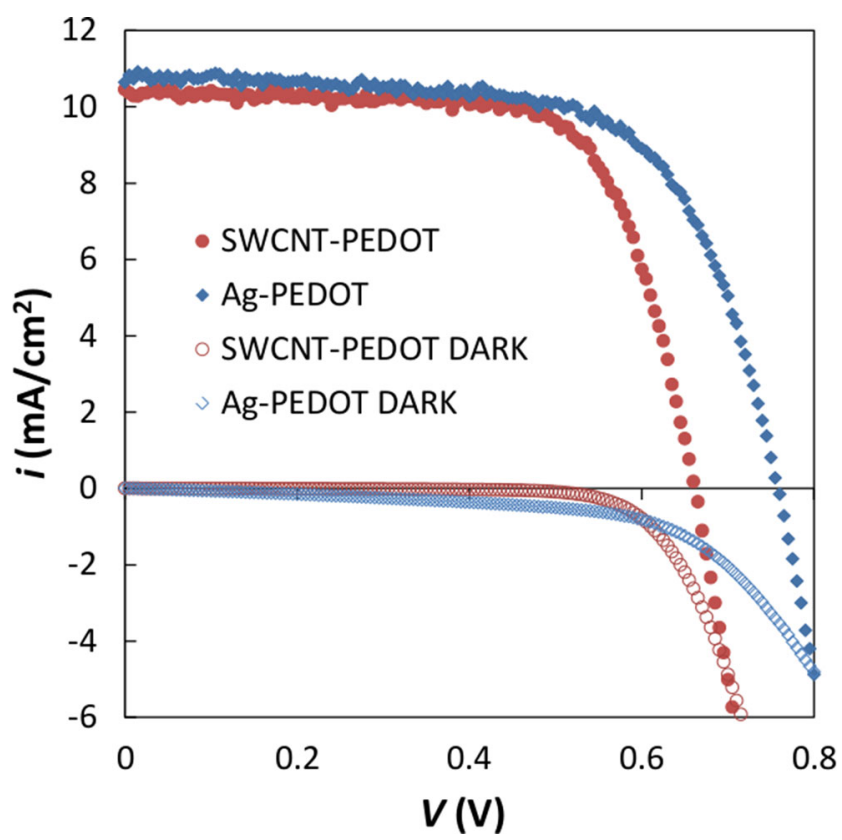

Fig. 3 Examples of typical $I-V$ curves of the DSCs with different counter electrodes and their dark current curves

cleaning would considerably reduce the cell manufacturing steps. This fact should however be verified in a long-term stability experiment, which is out of the scope of this study.

In the $I-V$ curves of the SWCNT-PEDOT cells, the onset of the diode-like recombination-related behavior occurs at lower voltages than in the Ag case (Fig. 3), which can be related to increased recombination from the $\mathrm{TiO}_{2}$ layer. This might be due to the fact that some SWCNTs, or fragments thereof, are detached from the SWCNT film, for instance during the densification process, and move onto the $\mathrm{TiO}_{2}$ layer, thereby accelerating the recombination between the $\mathrm{TiO}_{2}$ electrons and oxidized PEDOT.

Manseki et al. [22] observed a similar behavior for the carbon paste CE in the PEDOT-SSDSC, although much more pronouncedly: they obtained a much lower $V_{\mathrm{OC}}$ for the carbon-SSDSC than for the Ag-SSDSC. This result was ascribed to either the lower conductivity of the carbon paste or to the slower recombination at the photoelectrode in the AgSSDSC devices; according to this viewpoint, the latter effect is due to the fact that $\mathrm{Ag}$ forms a better (more uniform) contact with the PEDOT electrolyte layer.

The dark current curves of the SSDSCs are shown in Fig. 3. It can be seen that the dark current in the potential region 0 to $0.4 \mathrm{~V}$ is lower for the SWCNT-PEDOT cells than for the Ag-

Table 1 The $I-V$ parameters of the studied cells

\begin{tabular}{lllll}
\hline Cell type & $\eta(\%)$ & $j_{\mathrm{SC}}\left(\mathrm{mA} / \mathrm{cm}^{2}\right)$ & $V_{\mathrm{OC}}(\mathrm{V})$ & $F . F .(\%)$ \\
\hline PEDOT-SWCNT & 4.8 & 10.2 & 0.68 & 0.69 \\
PEDOT-Ag & 5.2 & 10.3 & 0.77 & 0.65 \\
\hline
\end{tabular}


PEDOT cells. In this region, the dark current originates from the contact of the polymer hole conductor with the FTO substrate, in case of accidental damage in some areas of the underlayer. This means that in the case of the SWCNT film CEs, the requirements for the $\mathrm{TiO}_{2}$ recombination-blocking underlayers might not be as stringent as in the silver case; the silver will tend to penetrate the PEDOT and $\mathrm{TiO}_{2}$ layers and contact the FTO glass.

The lifetime of the electrons in the $\mathrm{TiO}_{2}$ film as a function of the cell voltage obtained by the "tool-box" measurements is shown in Fig. 4. It can be seen that the lifetimes are similar for both types of cells, for voltage values differing by about $0.1 \mathrm{~V}$, which is also the difference in the $V_{\mathrm{OC}}$ in the two types of cells.

The short-circuit current density dependence on the incident light intensity is shown in the semi-logarithmic plot of Fig. 5. Since the dependence is linear, it can be concluded that there are no charge-transport limitation issues in either of the cell types.

Based on these measurements, it is thus difficult to give a precise explanation for the different $V_{\mathrm{OC}} \mathrm{S}$ obtained for the devices with the SWCNT and the Ag counter electrodes, which seems to stem from the difference in the CE properties. In this respect, a difference in the cell manufacturing process should be noted: the SWCNT-PEDOT-SSDSCs were not placed in the vacuum, unlike the Ag-PEDOT-SSDSCs during the silver evaporation. It is also worth noticing that the cells prepared for the present study are completely dry and do not contain either a high-boiling point solvent (ACN boiling point is $81.3^{\circ} \mathrm{C}$ ) or a redox mediator, unlike other high-efficiency PEDOT-SSDSCs previously described in the literature $[6,7]$. Some residual TBP might be present in the PEDOT film, but the volume fraction of TBP applied on the cell with the

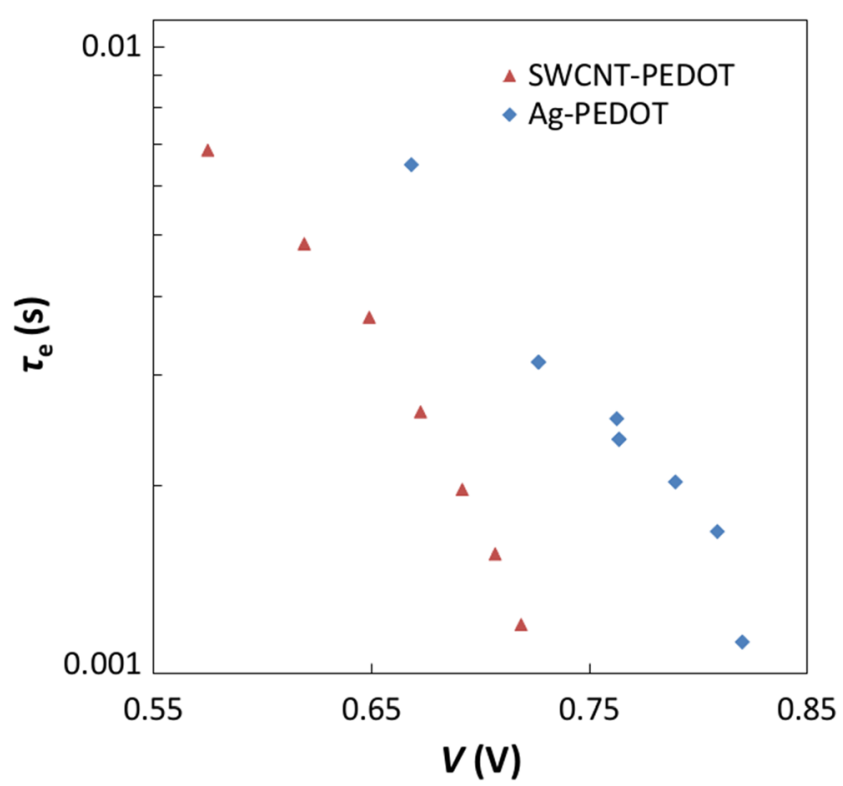

Fig. 4 The electron lifetime dependence on the open-circuit voltage

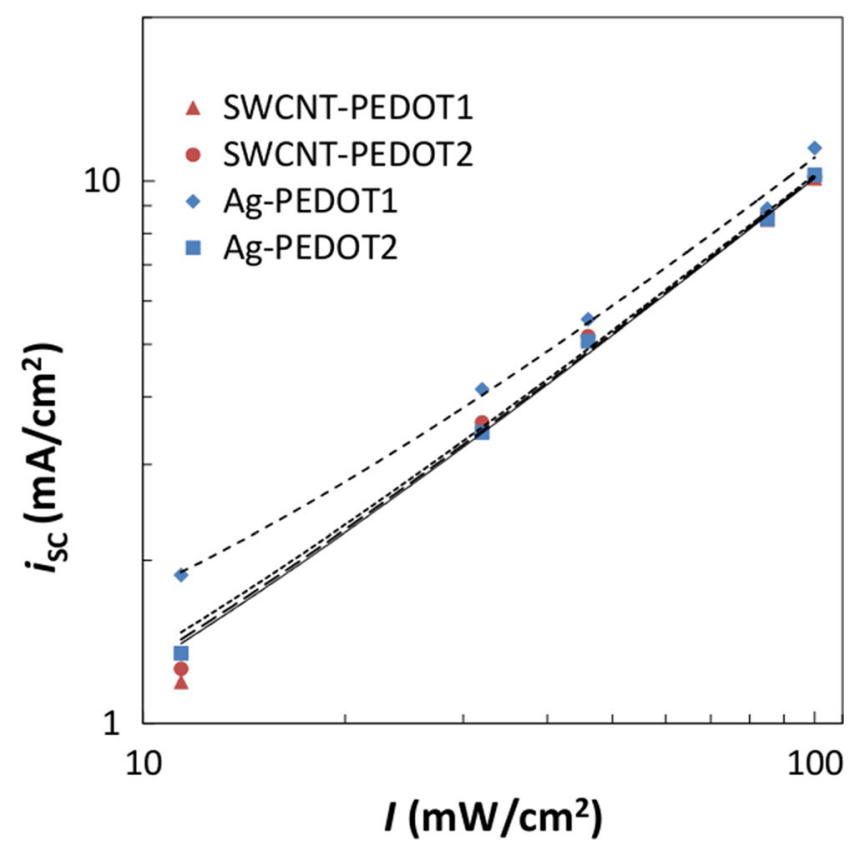

Fig. 5 The short-circuit current dependence of the light intensity

LiTFSI and TBP in ACN solution is only $3 \%$, and most of the solution is spun off from the cell already in the spincoating step. Part of the residual TBP is also adsorbed on the $\mathrm{TiO}_{2}$ film. Thus, we conclude that the amount of TBP is too small for making these PEDOT film non-dry.

\section{Conclusions}

The conducting silver layer of the PEDOT polymer-based solid-state dye solar cell was replaced with a presstransferred single-walled carbon nanotube film. Electrochemical removal of iron nanoparticles from the SWCNT films was not needed, unlike in the case when the SWCNT film is utilized as the counter electrode in a liquid electrolyte-based DSC. The efficiency of the SWCNT-SSDSC was $4.8 \%$ compared to $5.2 \%$ for the Ag-SSDSC. The performed measurements did not explain the slight efficiency difference, which stemmed from the approximately $0.1 \mathrm{~V}$ lower open-circuit voltage of the PEDOT-SWCNT cells. It is however believed that the potential difference is due to the slightly faster recombination from $\mathrm{TiO}_{2}$ because of some carbon nanotubes that detached from the counter electrode and deposited onto the $\mathrm{TiO}_{2}$.

Acknowledgments Financial assistance has been provided by the Swedish Energy Agency, the STandUP for Energy program, the Swedish Research Council (VR), the Knut and Alice Wallenberg Foundation, and the Aalto University project MIDECNB-E/FLEXCAP/MOPPI. K.A. acknowledges the Finnish Cultural Foundation and the Swedish Cultural Foundation in Finland for the Säätiöiden Post doc -pooli scholarships. J.Z. gratefully acknowledges the China Scholarship Council for a PhD study fellowship. 


\section{References}

1. O’Regan B, Grätzel M (1991) A low-cost, high-efficiency solar cell based on dye-sensitized colloidal $\mathrm{TiO}_{2}$ films. Nature 353:737-740

2. Asghar MI, Miettunen K, Halme J et al (2010) Review of stability for advanced dye solar cells. Energy Environ Sci 3:418-426

3. Bach U, Lupo D, Comte P et al (1998) Solid-state dye-sensitized mesoporous $\mathrm{TiO}_{2}$ solar cells with high photon-to-electron conversion efficiencies. Nature 395:583-585

4. Yang L, Xu B, Bi D et al (2013) Initial light soaking treatment enables hole transport material to outperform spiro-OMeTAD in solid-state dye-sensitized solar cells. J Am Chem Soc 135:7378-7385

5. Saito Y, Kitamura T, Wada Y, Yanagida S (2002) Poly(3,4ethylenedioxythiophene) as a hole conductor in solid state dye sensitized solar cells. Synth Met 131:185-187

6. Liu X, Zhang W, Uchida S et al (2010) An efficient organic-dyesensitized solar cell with in situ polymerized poly $(3,4-$ ethylenedioxythiophene) as a hole-transporting material. Adv Mater 22:150-155

7. Liu X, Cheng Y, Wang L et al (2012) Light controlled assembling of iodine-free dye-sensitized solar cells with poly $(3,4-$ ethylenedioxythiophene) as a hole conductor reaching $7.1 \%$ efficiency. Phys Chem Chem Phys 14:7098-7103

8. Kaskela A, Nasibulin AG, Timmermans MY et al (2010) Aerosolsynthesized SWCNT networks with tunable conductivity and transparency by a dry transfer technique. Nano Lett 10:4349-4355

9. Du Pasquier A, Unalan HE, Kanwal A et al (2005) Conducting and transparent single-wall carbon nanotube electrodes for polymerfullerene solar cells. Appl Phys Lett 87:1-3

10. Rowell MW, Topinka MA, McGehee MD et al (2006) Organic solar cells with carbon nanotube network electrodes. Appl Phys Lett 88:233506

11. Kymakis E, Stratakis E, Koudoumas E (2007) Integration of carbon nanotubes as hole transport electrode in polymer/fullerene bulk heterojunction solar cells. Thin Solid Films 515:8598-8600

12. Suzuki K, Yamaguchi M, Kumagai M, Yanagida S (2003) Application of carbon nanotubes to counter electrodes of dyesensitized solar cells. Chem Lett 32:28-29
13. Trancik JE, Barton SC, Hone J (2008) Transparent and catalytic carbon nanotube films 2008. Nano Lett 8:19-24

14. Aitola K, Kaskela A, Halme J et al (2010) Single-walled carbon nanotube thin-film counter electrodes for indium tin oxide-free plastic dye solar cells. J Electrochem Soc 157:B1831-B1837

15. Aitola K, Halme J, Feldt S et al (2013) Highly catalytic carbon nanotube counter electrode on plastic for dyesolar cells utilizing cobalt-based redox mediator. Electrochim Acta 111:206-209

16. Lee MM, Teuscher J, Miyasaka T et al (2012) Efficient hybrid solar cells based on meso-superstructured organometal halide perovskites. Science 338:643-647

17. Li Z, Kulkarni SA, Boix PP et al (2014) Laminated carbon nanotube networks for metal electrode-free efficient perovskite solar cells. ACS Nano 8:6797-6804

18. Habisreutinger SN, Leijtens T, Eperon GE et al (2014) Enhanced hole extraction in perovskite solar cells through carbon nanotubes. J Phys Chem Lett 5:4207-4212

19. Habisreutinger SN, Leijtens T, Eperon GE et al (2014) Carbon nanotube/polymer composites as a highly stable hole collection layer in perovskite solar cells. Nano Lett 14:5561-5568

20. Rong Y, Li X, Ku Z et al (2012) Monolithic all-solid-state dyesensitized solar module based on mesoscopic carbon counter electrodes. Sol Energy Mater Sol Cells 105:148-152

21. Xu M, Liu G, Li X et al (2013) Efficient monolithic solid-state dyesensitized solar cell with a low-cost mesoscopic carbon based screen printable counter electrode. Org Electron Phys Mater Appl 14:628-634

22. Manseki K, Jarernboon W, Youhai Y et al (2011) Solid-state dyesensitized solar cells fabricated by coupling photoelectrochemically deposited poly(3,4-ethylenedioxythiophene) (PEDOT) with silverpaint on cathode. Chem Commun 47:3120-3122

23. Gabrielsson E, Ellis H, Feldt S et al (2013) Convergent/divergent synthesis of a linker-varied series of dyes for dye-sensitized solar cells based on the D35 donor. Adv Energy Mater 3:1647-1656

24. Yang L, Zhang J, Shen Y et al (2013) New approach for preparation of efficient solid-state dye-sensitized solar cells by photoelectrochemical polymerization in aqueous micellar solution. J Phys Chem Lett 4:4026-4031 